\title{
Achieving Functionally Graded Material Composition Through Bicontinuous Mesostructural Geometry in Material Extrusion Additive Manufacturing
}

\author{
BRANT STONER,${ }^{1}$ JOSEPH BARTOLAI, ${ }^{1}$ DORCAS V. KAWEESA, ${ }^{1}$ \\ NICHOLAS A. MEISEL $, 2,3$ and TIMOTHY W. SIMPSON ${ }^{1}$ \\ 1.-Department of Mechanical and Nuclear Engineering, The Pennsylvania State University, \\ University Park, PA 16802, USA. 2.-School of Engineering Design, Technology, and Professional \\ Programs, The Pennsylvania State University, University Park, PA 16802, USA. 3.-e-mail: \\ nam20@psu.edu
}

\begin{abstract}
Functionally graded materials (FGMs) gradually change composition throughout their volume, allowing for areas of a part to be optimized for specific performance requirements. While additive manufacturing (AM) process types such as material jetting and directed energy deposition are capable of creating FGMs, design guidelines for varying the material composition in an FGM do not exist. This article presents a novel design solution for FGMs: creating the material gradient by varying the mesostructural size and thickness of bicontinuous, multi-material geometries. By using a bicontinuous structure, such as Schoen's gyroid surface or Schwarz's P and D surfaces, each component material exists as a continuous discrete structure, which allows FGMs to be fabricated by a wider range of AM processes. The gradient is created by varying the volume fraction occupied by the surface structure inside the part volume. This article explores the use of this technique to create FGMs with material extrusion AM. Properties of these bicontinuous structures are experimentally characterized and shown to outperform typical material extrusion FGMs.
\end{abstract}

\section{INTRODUCTION}

Functionally graded materials (FGMs) represent a new regime of composites comprised of two or more materials that continuously or discretely transition from one material to another throughout the bulk of the structure. First proposed in 1980 by Hirai et al. ${ }^{1}$ as a solution to meet the extreme thermal conditions on the skin of spacecraft, FGMs allow for a structure to exhibit multiple physical properties not possible within the same part made by conventional material processing techniques. There are currently three additive manufacturing (AM) processes capable of creating FGMs via different means: (1) material jetting, which uses dithering of material phases at the voxel level, ${ }^{2}(2)$ directed energy deposition (DED), which mixes multiple powder streams prior to deposition at a laser-induced melt-pool, ${ }^{3}$ and (3) material extrusion, which combines two separate thermoplastic filaments into a common melt pool before extruding the resulting composite onto a build platform. ${ }^{2}$
Both dithering and material mixing may result in isolated inclusions of one of the two component materials suspended within a matrix of the other material. When the part is mechanically loaded, the transfer of forces between the two component materials relies on traction at the matrix-inclusion interface. ${ }^{4}$ By relying on traction, the FGM cannot fully utilize the inherent mechanical strength of the included material, reducing its overall performance. If the FGM could be created in such a way that each component material would exist as a continuous entity, then this reliance on traction could be avoided.

This work presents a novel lattice structure design process that can create FGMs using dualmaterial extrusion, resulting in a bicontinuous mesostructure at the FGM interface. By creating a bicontinuous mesostructure, a discrete interface between two materials can be maintained, while the volume fraction of each material is varied. This creates a mechanical interlock between the two 
component materials, allowing for FGMs to be created using two immiscible materials because diffusion bonding is not required. This mechanical interlock can potentially increase the interfacial strength between FGMs produced using conventional dual-nozzle material extrusion printers.

\section{RELEVANT LITERATURE}

\section{Functionally Graded Materials}

The development of FGMs has struggled because of two main challenges: (1) the challenge of designing FGMs and (2) representing the material distribution in a meaningful way. Two main approaches, voxel-based models and function-based models, have been explored to address these two challenges. ${ }^{5}$ Voxel representation of FGMs defines the material composition of discrete elements within the structure. ${ }^{6}$ Alternatively, function-based models utilize global and local equations to define the material distribution of a part. While very fine control of the material distribution can be achieved by either method, fitting of gradient functions to complex geometries presents a challenge in determining the compatibility requirements between regions.

Garland and $\mathrm{Fadel}^{7}$ carried out an experimental analysis of material extrusion FGMs using the commercially available Big Builder Dual-Feed extruder. The system uses an extrusion head that feeds two filaments into a novel melt chamber to mix materials during the building process. Using two filaments of different colors, a color gradient can be created by varying the feed rate of the two filaments. While Garland and Fadel concluded that there was insufficient material mixing in the dualfeed extruder to reliably control material composition, they do present dual-feed material extrusion $\mathrm{AM}$ as a useful tool to visualize FGMs.

\section{Lattice Structures}

Lattice structures are formed by repeating a base geometry to form a bulk structure. The unit cell is the smallest nonrepeating constituent of the lattice. Combining unit cells in a repeated fashion forms the mesostructure of a component. Unit cell designs are most often created via either primitive-based Boolean methods ${ }^{8}$ or implicit methods ${ }^{9}$ and have been shown to be effective in impact-absorbing structures, ${ }^{10}$ weight reduction, ${ }^{11}$ and mimicking biological structures. ${ }^{12}$ Graded lattice structures are designed to allow for differing properties throughout the bulk structure, allowing the lattice structure to mimic the performance gains of FGMs. This is accomplished by varying parameters of the unit cell such as the strut diameter, number of struts, or unit cell morphology to produce a heterogeneous lattice structure. ${ }^{13}$

\section{METHODOLOGY}

This work proposes a novel method for creating lattice structures and FGMs by combining both primitive-based Boolean and implicit lattice generation methods. This process is summarized in Fig. 1. The input part geometry is first digitized into voxels. An implicitly generated graded lattice structure is then applied to the part geometry. This lattice structure is then combined with the original geometry. The volume occupied by the graded lattice structure and the resulting Boolean volume are each assigned one of the two FGM component materials. Finally, the two component material volumes are combined and fabricated as a single functionally graded multi-material part.

Unique to this process, changes in the lattice geometry are used to produce variations in material compositions. The lattice structure is generated implicitly, by using a triply periodic minimal surface (TPMS) defined by a closed-form equation. One period of the TPMS forms the unit cell for the lattice structure. The four TPMSs explored are Schoen's gyroid, Schwarz's P and D surfaces, and Lidin's Lidinoid. ${ }^{9}$ These four TPMSs are defined by Eqs. 1, 2,3 , and 4 .

Schoen's gyroid: ${ }^{9}$

$\sin (x) \cos (y)+\sin (y) \cos (z)+\sin (z) \cos (x)=0$

Schwarz P surface: ${ }^{9}$

$$
\cos (x)+\cos (y)+\cos (z)=0
$$

Schwarz D surface: ${ }^{9}$

$$
\begin{aligned}
& \sin (x) \sin (y) \sin (z)+\sin (x) \sin (y) \cos (z) \\
& \quad+\cos (x) \sin (y) \cos (z)+\cos (x) \cos (y) \sin (z)=0
\end{aligned}
$$

$$
\begin{aligned}
& \text { Lidinoid: }{ }^{14} \\
& \begin{array}{l}
\frac{1}{2}[\sin (2 x) \cos (y) \sin (z)+\sin (2 y) \cos (z) \sin (x) \\
\quad+\sin (2 z) \cos (x) \sin (y)]-\frac{1}{2}[\cos (2 x) \cos (2 y) \\
\quad+\cos (2 y) \cos (2 x)+\cos (2 z) \cos (2 x)]+0.15=0
\end{array}
\end{aligned}
$$

The FGM is created by assigning one material to the surface defined by one of the four surface equations and the other material to the surrounding volume. The material gradient is created by varying the thickness of the surface between the userdefined gradient endpoints. If a point $\{x, y, z\}$ satisfies one of the minimal surface equations, then that point lies on the surface defined by that equation. By definition, these surfaces have zero thickness; however, adjusting the surface equation so that it is evaluated as an inequality allows a nonzero thickness surface to be defined. Scaling terms 


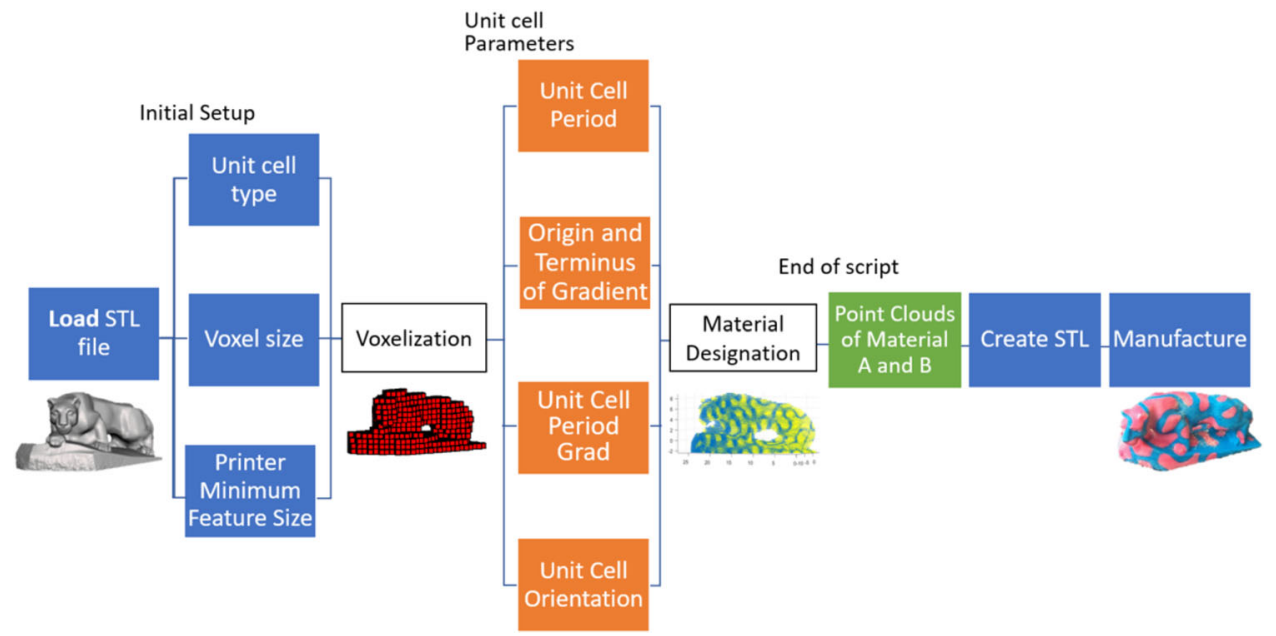

Fig. 1. Bicontinuous, graded mesostructure design workflow.

are also added to define the period of the minimal surface. An example of this is shown for the gyroid in Eq. 5.

$$
\left|\sin \left(\frac{2 \pi}{P} x\right) \cos \left(\frac{2 \pi}{P} y\right)+\sin \left(\frac{2 \pi}{P} y\right) \cos \left(\frac{2 \pi}{P} z\right)+\sin \left(\frac{2 \pi}{P} z\right) \cos \left(\frac{2 \pi}{P} x\right)\right| \leq t
$$

where $x, y$, and $z$ are the Cartesian coordinates of any location in the part, $P$ is the period of the minimal surface, and $t$ is a unitless thickness value.

Each point of the discretized model is tested against this inequality for material designation. If the inequality is true, then material A is assigned; otherwise, material $\mathrm{B}$ is assigned. $t$ varies from 0 to a maximum value that is inherent in the TPMS used. In the case of the gyroid, this maximum value is 1.5 . In this work, the thickness value is scaled linearly between the minimum and maximum values across the material gradient region. Because each of the TPMSs studied in this work is defined by continuous equations, surface thickness and period parameters can be changed not only across the material gradient region but also within each unit cell. This differs from traditional lattice structures that can only implement changes at unit cell boundaries. Using this method, a nearly infinitely variable lattice structure can be created.

\section{PARAMETER DEVELOPMENT}

As shown in Fig. 1, the initial setup consists of loading the STL file and then choosing the unit cell type, voxel size, and AM machine minimum feature size. In this work, the voxel size is set to half of the minimum feature size or intended layer thickness, whichever is smaller, to minimize stair-stepping effects. Unit cell design follows after this initialization, which includes defining both the positive space for the first material phase and the inverse space for the second material phase (see Fig. 2).

The explicit closed-form nature of the selected TPMS allows for easy manipulation of four unit cell parameters to fit the desired application: (1) period of the unit cell, (2) origin and terminus of the gradient, (3) unit cell period gradient, and (4) unit cell orientation. Figure 3 shows the effects of changing these parameters independently. A reference geometry is shown in Fig. 3a to illustrate the effects of the parameter changes.

\section{Unit Cell Period}

The first parameter to be set is the period of the TPMS; this controls the size of the unit cell and affects the granularity of the functional gradient between the two materials. As shown in Fig. 3c, a decrease in the period length from $10 \mathrm{~mm}$ to $5 \mathrm{~mm}$ gives a better approximation of a functional gradient. As the period length approaches zero, the bicontinuous mesostructure becomes a conventional functional gradient.

\section{Gradient Bounds}

The gradient origin and terminus planes are determined by user-generated origin and terminus points. Planes are generated through these points using the vector between them as the normal vector for each of the two planes. These endpoints are in the form of Cartesian coordinates $\{x, y, z\}$ and can be placed anywhere in the structure. The region opposite the normal for the origin plane is assigned Material A, and the region opposite the normal for the terminus plane is assigned Material B. In Fig. 3d the same $40-\mathrm{mm}$ gradient length was used, but a $\{0.7,0.7,0.3\}$ vector was used as the normal for the origin and terminus plans.

\section{Unit Cell Period}

The concept of functional gradients also extends to the periods of the unit cell, which can be graded through the bulk of a part as seen in Fig. 3e. The size of the unit cell changes depending on the starting and ending conditions set by the user by indicating the initial and end period lengths. The 

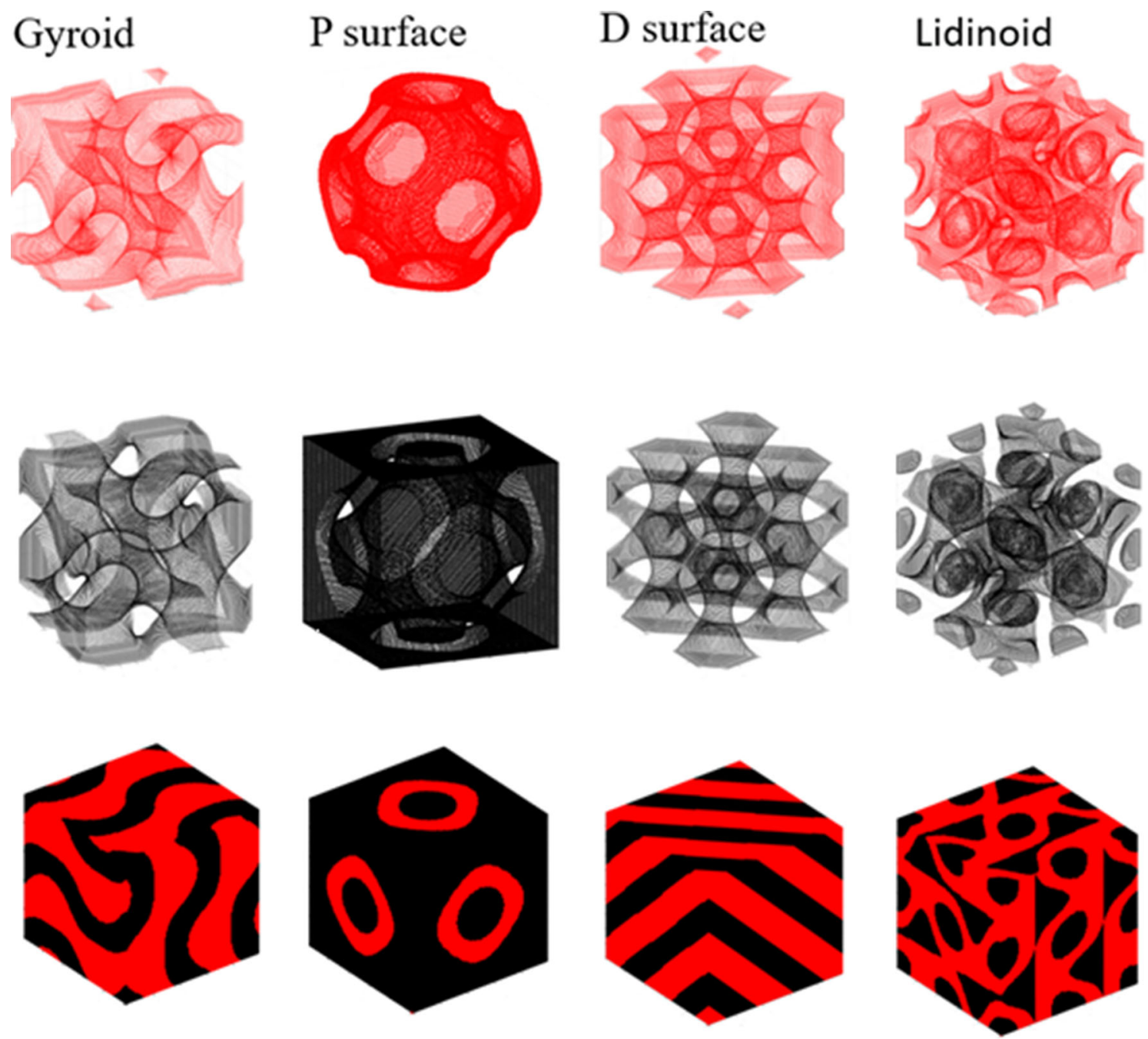

Fig. 2. Examples of material a, material b, and combined a and b unit cells for the gyroid, P surface, D surface, and Lindinoid.

(a)

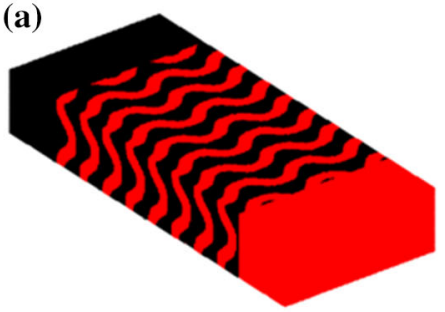

(d)

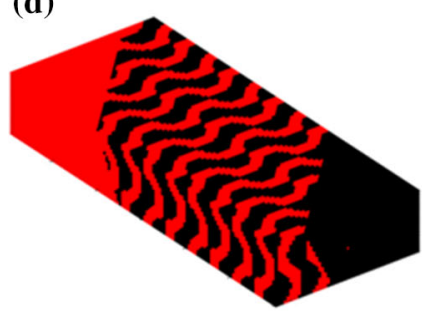

(b)

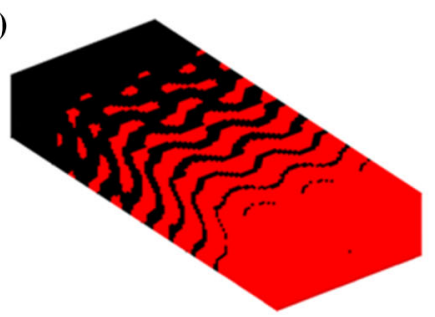

(e)

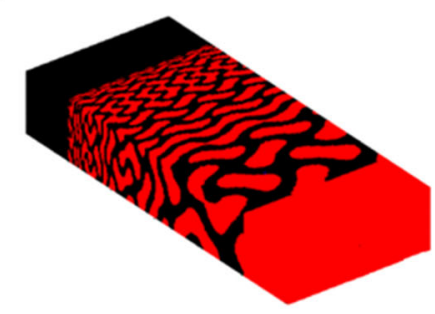

(c)

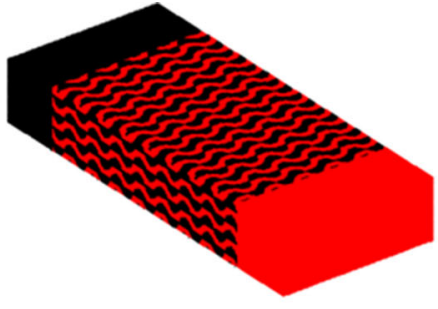

(f)

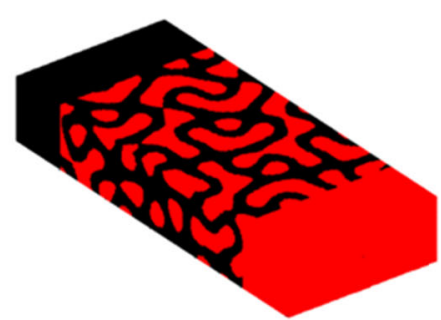

Fig. 3. Possible mesostructure transformations that show (a) the base mesostructure, (b) graded thickness, (c) a reduced period, (d) altered origin and terminus planes, (e) a graded period, (f) a rotated gyroid unit cell. 


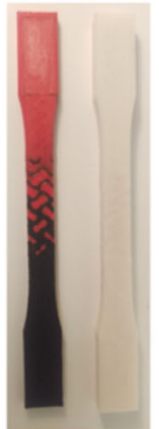

(a)

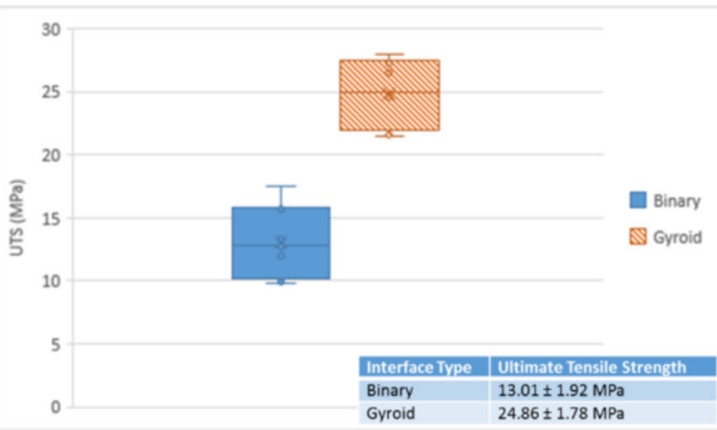

(b)

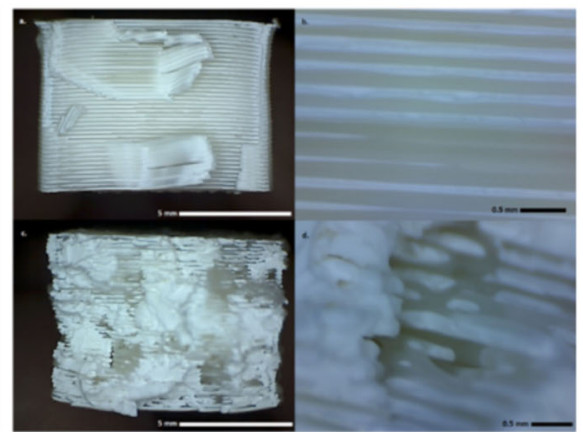

(c)

Fig. 4. (a) The FGM component used in testing, (b) tensile test results, and (c) comparison of binary fracture surface (top) to gyroidal surface (bottom).

period of the unit cell in Fig. 3e shifts from $5 \mathrm{~mm}$ to $15 \mathrm{~mm}$ along the length of the gradient. A potential application for this is to incorporate stiffness matching between the two component lattices into the design process. By developing a relationship between the period length and the resulting modulus, regions of a component could be tailored to a specific application.

\section{Unit Cell Orientation}

Lastly, the orientation of the unit cell can be modified so that it is aligned with a specific vector $\{x$, $y, z\}$. The unit cell orientation is changed by the user by defining the direction of the unit cell $x$ axis relative to the part's local coordinate frame. The orientation of the unit cell could have a significant impact on the mechanical properties of the mesostructure, such as strength and compliance. A $\{1,1,1\}$ vector was used to orient the unit cell in Fig. 3f.

\section{Material Assignment and Manufacturing}

Once all unit cell parameters have been defined, then material types are assigned to the vowelized file based on the TPMS equations. A Comma Separated Variable (CSV) point cloud is generated to represent the surface of each component material. Avizo (FEI Co., Hillsboro, OR, USA) was used to convert the point cloud to an STL file. Unfortunately, the meshing process often does not create a single continuous "watertight" surface. Magics (Materalise NV, Leuven, Belgium) is used to fix the meshed STL files, align them and save them as one STL. This STL is then loaded into Slic3r, which can identify independent shells and generate specimen production toolpaths.

\section{PRELIMINARY MECHANICAL TESTING}

To understand the impact of this novel FGM process on part performance, mechanical property tests were performed. Because the mechanical properties of Schoen's gyroid have been previously evaluated in tension ${ }^{15}$ and compression, ${ }^{16}$ the gyroid mesostructure strength was evaluated first. Specimens consisting of both a graded gyroidal mesostructure and a binary material interface were tested in uniaxial tension. To eliminate as many experimental variables as possible, the same 1.75$\mathrm{mm}$ diameter acrylonitrile-butadiene-styrene (ABS) filament feedstock was used for both components of the graded mesostructure specimens and both halves of the binary interface specimens. To ensure that the tensile specimens included at least one full period of the gyroid along each of the local coordinate axes, an ASTM D638 Type $\mathrm{I}^{17}$ tensile specimen was modified to have a thickness of $10 \mathrm{~mm}$ (see Fig. 4a). The binary interface specimens were also produced in this geometry, ensuring a one-to-one comparison of the mechanical response of the two types of interface. All specimens were produced in XYZ orientation on a Mendel Max 3 machine (Maker's Tool Works, OK, USA) with an extrusion temperature of $235^{\circ} \mathrm{C}$ and a deposition surface temperature of $120^{\circ} \mathrm{C}$.

Tensile test results for the binary and gyroid specimens show ultimate tensile strengths of $13.01 \pm 1.92 \mathrm{MPa}$ and $24.86 \pm 1.78 \mathrm{MPa}$, respectively (see Fig. 4b), and 95\% confidence intervals were calculated using the Student's $t$ distribution for a sample size of 10 . As shown, the mean graded mesostructure interface was $91 \%$ stronger than the mean binary interface. A comparison of the fracture surfaces of the two types of interface shows a clear planar fracture surface in the binary interface specimens (see Fig. 4c). The gyroidal interface specimen fracture surface is much more complex and demonstrates more crazing deformation. This, along with the increased strength, is an indication of better fusion at the fracture surface, which is evidence of improved integration of the two component structures.

\section{CONCLUSION AND FUTURE WORK}

A bicontinuous graded mesostructure material interface has been shown to be much stronger than a binary material interface, producing parts with a 
91\% greater ultimate strength. The novel method for creating FGMs and lattice structures based on the TPMS presented shows great promise for implementation into end-use FGM parts produced by material extrusion AM and other AM processes. While these are only preliminary results, we are optimistic about the possible advantages of TPMSbased bicontinuous material interfaces. Due to the limited scope of the testing thus far, a large portion of the graded mesostructure design space remains unexplored. Future experiments will test the interfaces of two different thermoplastic polymers. Differences in the four TPMS unit cells will also be evaluated, along with advantages and disadvantages of assigning materials with a large difference in stiffness from both component structures. Effects of changes in each of the TPMS unit cell design parameters will also need to be characterized.

\section{REFERENCES}

1. S. Masayuki Niino, M. Akio Suzuki, H. Toshio, I. Takamori, R. Watanabe, S. Tohru Hirano, and I. Nobuhito Kuroishi, Method of Producing a Functionally Graded Material (1988), pp. 1-5.

2. ISO/ASTM International, ISO/ASTM Standard 52900:2015(E), 1 (2015).
3. M.L. Griffith, L.D. Harwell, J.T. Romero, E. Schlienger, C.L. Atwood, and J.E. Smugeresky, in Proceedings of the 8th Solid Freeform Fabrication Symposium, vol. 387 (1997).

4. J. Aboudi, M.J. Pindera, and S.M. Arnold, Compos. Part B Eng. 30, 777 (1999).

5. X.Y. Kou and S.T. Tan, CAD Comput. Aided Des. 39, 284 (2007).

6. E.L. Doubrovski, E.Y. Tsai, D. Dikovsky, J.M.P. Geraedts, H. Herr, and N. Oxman, CAD Comput. Aided Des. 60, 3 (2015).

7. A. Garland and G. Fadel, J. Mech. Des. 137, 111407 (2015).

8. D.S. Nguyen and F. Vignat, in IEEE International Conference on Industrial Engineering and Engineering Management, vol. 966 (2016).

9. A.H. Schoen, Nasa Tech. Note D-5541. 92 (1970).

10. J. Brennan-Craddock, D. Brackett, R. Wildman, and R. Hague, J. Phys. Conf. Ser. 382, 12042 (2012).

11. C. Yan, L. Hao, A. Hussein, P. Young, and D. Raymont, Mater. Des. 55, 533 (2014).

12. X. Li, J. He, W. Zhang, N. Jiang, and D. Li, Materials (Basel). 9, 1 (2016).

13. Y. Tang, A. Kurtz, and Y.F. Zhao, CAD Comput. Aided Des. 69, 91 (2015).

14. S. Lidin and S. Larsson, J. Chem. Soc. Faraday Trans. 86, 769 (1990).

15. I. Maskery, C. Tuck, A.O. Aremu, I. Maskery, C. Tuck, I.A. Ashcroft, R.D. Wildman, and R.I. Hague, in International Solid Freeform Fabrication Symposium, vol. 1238 (2014).

16. Z. Qin, G.S. Jung, M.J. Kang, and M.J. Buehler, Sci. Adv. 3,1 (2017).

17. ASTM International, ASTM Standard D638-10. 1 (2013). 